\title{
Observational case-control study of non-invasive ventilation in patients with ARDS
}

\author{
G. Domenighetti, A. Moccia, R. Gayer
}

\begin{abstract}
Observational case-control study of noninvasive ventilation in patients with ARDS. G. Domenighetti, A. Moccia, R. Gayer.

Background. The application of non-invasive pressure support ventilation (NIPSV) in patients with acute lung injury or ARDS remains controversial despite recent promising results. Data in rather homogeneous ARDS groups is lacking.

Objective. To compare the outcome of NIPSV-treated patients satisfying the diagnostic criteria for primary (pulmonary) ARDS (ARDS $)_{p}$ ) and presenting without distant organ failures at admission, with those of a matched control group treated in the same ICU with endotracheal mechanical ventilation (ETMV).

Methods. We applied NIPSV in 12 immunocompetent and collaborative patients who met the above cited criteria. NIPSV failure rate, short-term oxygenation, length of stay, mortality rate and complications were analyzed and com-
\end{abstract}

pared with a control group of 12 intubated $\mathrm{ARDS}_{\mathrm{p}}$-patients matched for age, $\mathrm{SAPS}$ II, $\mathrm{PaO}_{2} / \mathrm{FiO}_{2}$ and $\mathrm{pH}$ at admission.

Results. NIPSV failed in 4 patients developing distant organ failures. Compared to the ETMV control group, NIPSV success patients had reduced cumulative time on ventilation $(p=0.001)$ and length of ICU stay $(p=0.004)$. After the first 60' of ventilation, oxygenation improved more in the NIPSV than in the ETMV group $(146 \pm 52$ $\mathrm{mmHg}$ vs $109 \pm 34 \mathrm{mmHg} ; p=0.05$ ). The overall ICU mortality rate did not differ significantly between the groups but tended to be higher in the NIPSV group.

Conclusions. In ARDS patients without distant organ failures at admission and during the disease course, NIPSV might be a suitable alternative to invasive ventilation; however, the real effects on outcome of NIPSV applied to stable homogeneous subgroups of ARDS patients merit further investigations in randomised studies.

Monaldi Arch Chest Dis 2008; 69: 1, 5-10.

Keywords: Noninvasive ventilation, Conventional mechanical ventilation, ARDS, Primary ARDS.

Correspondence: Dr. G. Domenighetti, Regional Hospital "La Carità", Multidisciplinary Intensive Care Unit, Via Ospedale 14, 6600 Locarno, Switzerland; e-mail: guido.domenighetti@eoc.ch

\section{Introduction}

The application of non-invasive pressure support ventilation (NIPSV) in patients with hypoxemic acute respiratory failure (HARF) unrelated to COPD still remains controversial despite of promising results [1-3]. So far, published clinical randomized or non-randomised studies on NIPSV in non-COPD-related HARF have focused on single etiologies (cardiogenic pulmonary edema, community-acquired pneumonia or both $[2,3,4,5$, $6]$ ) or on mixed populations with a variety of diagnoses $[1,7,8]$. In these latter studies, the clinical outcome according to the underlying nature of HARF was seldom assessed [7, 8]. Interestingly enough, some of the latter investigations as well as others [9] have included noninvasively ventilated patients with a diagnosis of ARDS [10]. Overall, 185 patients may be considered from these various studies, with a global NIPSV failure rate of $42 \%$ and a mortality rate of $29 \%$ [11]. More recently, Antonelli and co-workers reported a prospective survey of NIPSV in 147 ARDS patients in which $46 \%$ failed and were intubated. Predictors used to see whether there is a need for intubation included greater age, higher Simplified Acute Physiology Score (SAPS II) and the inability to improve
$\mathrm{PaO}_{2} / \mathrm{FiO}_{2}$ after one hour of non-invasive ventilation [12]. In a previous study performed in patients with severe community-acquired pneumonia, we found that a co-existing bacteremia associated with multiple organ dysfunction was predictive of a high NIPSV failure rate [5]. We speculated that NIPSV might also constitute an effective method for treating HARF in patients with a diffuse pulmonary injury satisfying the diagnostic criteria for a primary ARDS (ARDSp) and admitted without extra-pulmonary organ failures. To address this question, we analysed the application of this technique in a cohort of relatively homogeneous patients presenting primarily with a diffuse pneumonia as a cause of acute respiratory distress syndrome and compared their outcomes with those of a matched control group previously treated in the same ICU with endotracheal mechanical ventilation (ETMV).

Material and methods

\section{Patient selection}

Between February 2002 and October 2004, all consecutive adult patients with early primary ARDS were considered potentially eligible for the 
study. They presented with the classical criteria for invasive ventilation: (a) severe dyspnea at rest, (b) respiratory rate $\geq 30$ breaths/min and (c) diagnostic criteria for ARDS, according to the American European consensus conference on ARDS definition [10]. Among 29 patients admitted with this diagnosis, 17 required intubation for altered mental status, inability to manage secretions, haemodynamic instability, trauma or multiple organ failures (acute or exacerbated renal failure, cardiovascular insufficiency, altered coagulation, liver failure and central nervous system disorders). Twelve patients, fulfilling the following inclusion criteria for NIPSV, were selected: hemodynamic stability (systolic blood pressure $\geq 90 \mathrm{mmHg}$ or mean systemic pressure $\geq 65 \mathrm{mmHg}$ ) without vasopressors, lack of severe ventricular arrhythmias or myocardial ischemia, good cooperation (Glasgow Coma Scale $\geq 12$ ) and anamnestic lack of claustrophobia; ability to clear respiratory secretions; no significant metabolic and/or respiratory acidosis $\left(\mathrm{pH}_{\mathrm{a}}\right.$ $\geq 7.30$ ); immunocompetence; and no extrapulmonary organ dysfunction. Written informed consent was obtained from all non-invasively ventilated patients and the study was approved by the cantonal ethics committee.

\section{NPSV and criteria for endotracheal intubation}

Immediate standard management in the Emergency Room, ward or ICU (before NIPSV) included high-flow oxygen administration with high concentration masks the achievement of, where possible, a level of $\mathrm{SpO}_{2} \geq 88 \%$ antibiotic administration following local and ATS guidelines [13], chest physiotherapy if practicable and fluids. Inhaled bronchodilators and steroids were used at the discretion of the attending physician. The criteria for diagnosing pneumonia were those proposed by the ATS $[13,14]$. We used masks with an adjustableair soft-cushion covering for the mouth and nose (Vital Signs Inc. Barnham, UK). The mask was adjusted to avoid air leaks and connected to an ICU ventilator (SERVO-I Siemens Elema, Uppsala, Sweden) set in the flow-cycled pressure support mode. The servo-I ventilator permits a set on cycle from inspiration to expiration; to avoid unwarranted prolonging of the mechanical insufflations into the patient-initiated expiration, the ventilator was individually set for the patient's best expiratory trigger sensitivity. This allowed the reduction of episodes of asynchronies. Moreover, a back-up function was available with each assisted mode of ventilation in case of apnea. The initial ventilatory settings were PEEP $5 \mathrm{~cm} \mathrm{H}_{2} \mathrm{O}$ and pressure support 8 to $12 \mathrm{~cm} \mathrm{H}_{2} \mathrm{O}$. Subsequently, the nurse and attending physician adjusted pressure support (to achieve an exhaled tidal volume of 7 to $10 \mathrm{ml} / \mathrm{kg}$ IBW), PEEP-level and $\mathrm{FiO}_{2}$ based on tolerance; the goal was achievement of a respiratory rate $(\mathrm{RR})<25$ breaths/min and an oxygen saturation between $88 \%$ and $92 \%$ as measured by pulseoximetry. Major criteria for endotracheal intubation and NIPSV failure, were based on those defined by Brochard et al. [15]; these included respi- ratory arrest, respiratory pauses with loss of consciousness or gasping for air, psychomotor agitation making nursing care impossible and requiring sedation, a heart rate below 50 beats per minute with loss of alertness, and hemodynamic instability with systolic arterial blood pressure below 70 $\mathrm{mmHg}$. Additional criteria for intubation and NIPSV failure were: clinical signs of exhaustion, RR above the admission value after 2 hours of NIPSV, failure to maintain an $\mathrm{SpO}_{2} \geq 88 \%$, significant metabolic and/or respiratory acidosis $\left(\mathrm{pH}_{\mathrm{a}}\right.$ $\leq 7.30$ ) and changes in mental status linked to respiratory impairment. The presence of one major criterion was considered to indicate the need for intubation and mechanical ventilation; the presence of two additional criteria after two hours of NIPSV was considered to indicate the need for early intubation. With improved clinical status and ventilatory parameters ( $\mathrm{RR}, \mathrm{SpO}_{2}$, gas exchange values), NIPSV was virtually continuously maintained for the first 24 hours. Once $\mathrm{FiO}_{2}$ was 0.5 or less and PEEP was $5 \mathrm{~cm} \mathrm{H}_{2} \mathrm{O}$, and the patient clinically improved, inspiratory pressure support was reduced stepwise in increments of $2 \mathrm{~cm} \mathrm{H}_{2} \mathrm{O}$. Noninvasive ventilation was discontinued and considered successful when unassisted spontaneous breathing was sustained for 24 consecutive hours with a $\mathrm{pH} \geq 7.35$ and $\mathrm{SpO}_{2} \geq 90 \%$ with breathing mask oxygen at $\mathrm{FiO}_{2} \leq 0.4$.

\section{Controls (ETMV)}

Controls were all ARDSp patients selected from a group of 30 ARDS patients admitted in the previous two years to the same ICU who were treated with endotracheal intubation (EI) and mechanical ventilatory support within the first 6 hours of ICU admission. Exclusion criteria included patients who had received an NIPSV trial prior to intubation as well as extrapulmonary ARDS forms; we also excluded those primary ARDS patients who were immediately intubated before being transferred to the ICU. This was because this group of patients presented to a large extent with extra-pulmonary organ dysfunctions and an higher severity score (SAPS II). As a result, 18 historical patients with primary ARDS were eligible as controls from the database. Then, 12 immunocompetent and haemodynamically stable controls [9 patients with diffuse pneumonia and 3 patients with toxic-mediated ARDS] were finally selected according to the following matching criteria: (1) age \pm 8 years; (2) SAPS II, assessed within the first 24 hours after ICU-admission, \pm 6 points; (3) $\mathrm{PaO}_{2} /$ $\mathrm{FiO}_{2}$ ratio before institution of EI, \pm 20 points; and (4) $\mathrm{pH}$ before institution of EI, \pm 0.02 . Criteria for diagnosing pneumonia were those proposed by the ATS [13, 14]. The ventilators used were the same as those described for the NIPSV group. After intubation, patients were ventilated with a lung protective strategy according to the ARDS Network criteria [16]. Our criteria regulating the decrease of support and the discontinuation of mechanical ventilation have been previously published [17]. 


\section{Physiological measurements}

The following data was obtained at study entry: complete chemical and haematological tests, diagnostic microbiological tests (suctioned airway secretions, BAL when indicated and practicable, urine and blood). The baseline physiological assessment before starting NIPPV, included SAPS II, the Glasgow Coma Scale, complete vital parameters, $\mathrm{SpO}_{2}$ and complete blood gas analysis. Highflow mask systems were used to deliver oxygen at high concentrations before institution of NIPSV. Delivered $\mathrm{FiO}_{2}$ was approximately estimated using a calculation table. In order to better corroborate the oxygenation, $\mathrm{FiO}_{2}$ was estimated on a graph considering $\mathrm{O}_{2}$ flow and the spontaneous minute volume ventilation determined by a Wright spirometer. These variables were monitored and again recorded after 60 minutes and regularly as long as necessary. Blood gas analysis was assessed immediately (Blood Gas Analysator, AVL Medical Systems, Schaffhausen, Switzerland). Complications associated with NIPSV and ETMV were recorded.

\section{Data analyses}

Results are reported as mean \pm SD. Data on figures is graphically depicted as a box-Whysker diagram. Comparisons of normally distributed variables between cases and controls were done using unpaired $t$-tests. Qualitative variables were analysed using Mc Nemar's test. Subgroup analysis involving comparisons between unmatched groups were made using unpaired $t$-tests, chisquare or Fisher's exact tests. $P$ values lower than 0.05 were considered to be statistically significant.

\section{Results}

The matching variables, other characteristics at enrollment and the outcomes of the two groups are listed in table 1. Underlying cause were mainly diffused community-acquired pneumonia in 15 patients or diffuse nosocomial pneumonia in 5 patients. Although all patients in both groups had blood and airway secretions taken for cultures purposes, a specific microbiological diagnosis was established in 6 patients in the NIPSV group and in

Table 1. - Comparison between noninvasive pressure support ventilation (NIPSV) and conventional endotracheal mechanical ventilation (ETMV) groups

\begin{tabular}{|c|c|c|c|c|}
\hline & $\begin{array}{l}\text { NIPSV } \\
(n=12)\end{array}$ & $\begin{array}{c}\text { ETMV } \\
(\mathrm{n}=12)\end{array}$ & $\underset{\text { value }}{\mathbf{p}}$ & $\begin{array}{c}\% \text { of } \\
\text { accordance }\end{array}$ \\
\hline \multicolumn{5}{|l|}{ MATCHING CRITERIA } \\
\hline AGE (years) & $66 \pm 8$ & $64 \pm 6$ & 0.45 & 94 \\
\hline SAPS II & $44 \pm 12$ & $47 \pm 10$ & 0.41 & 92 \\
\hline $\mathrm{PaO}_{2} / \mathrm{FiO}_{2}(\mathrm{mmHg})$ & $104 \pm 42$ & $96 \pm 42$ & 0.67 & 93 \\
\hline $\mathrm{pH}$ & $7.39 \pm 0.07$ & $7.38 \pm 0.09$ & 0.52 & 98 \\
\hline \multicolumn{5}{|l|}{ CHARACTERISTICS } \\
\hline Male / female & $5 / 7$ & $9 / 3$ & 0.21 & \\
\hline \multicolumn{5}{|l|}{ Cause of ARDS } \\
\hline - Pneumonia (n) & 11 & 9 & 0.34 & \\
\hline - Community-acquired & 8 & 7 & & \\
\hline - Hospital-acquired & 2 & 3 & & \\
\hline - Other* (n) & 1 & 3 & & \\
\hline Chronic comorbidities $^{\Delta}(\mathrm{n})$ & $1.2 \pm 0.5$ & $1.4 \pm 0.6$ & 0.15 & \\
\hline RR before ventilation (breaths/min) & $34 \pm 4$ & $36 \pm 6$ & 0.15 & \\
\hline HR before ventilation (beats/min) & $111 \pm 18$ & $109 \pm 21$ & 0.18 & \\
\hline MAP (mmHg) & $87 \pm 12$ & $82 \pm 13$ & 0.1 & \\
\hline $\mathrm{PaO}_{2}$ before ventilation $(\mathrm{mmHg})$ & $57 \pm 9$ & $56 \pm 11$ & 0.1 & \\
\hline $\mathrm{PaCO}_{2}$ before ventilation $(\mathrm{mmHg})$ & $37 \pm 9$ & $40 \pm 14$ & 0.8 & \\
\hline $\mathrm{pH}$ before ventilation & $7.39 \pm 0.07$ & $7.37 \pm 0.09$ & 0.4 & \\
\hline Max. PEEP-level used $\left(\mathrm{cm} \mathrm{H}_{2} \mathrm{O}\right)$ & $6 \pm 0.8$ & $10 \pm 2.5$ & 0.0001 & \\
\hline \multicolumn{5}{|l|}{ OUTCOMES } \\
\hline ICU Mortality (n; \%) & $5(42)$ & $3(25)$ & 0.69 & \\
\hline LOS ICUa (hrs) & $106 \pm 60$ & $404 \pm 253$ & 0.004 & \\
\hline LOS ICUb (hrs) & $184 \pm 139$ & $404 \pm 253$ & 0.023 & \\
\hline
\end{tabular}

Data are expressed as mean \pm SD.

NIPSV = noninvasive pressure support ventilation; ETMV = endotracheal mechanical ventilation; SAPS II = simplified acute physiology score; $\Delta=$ chronic heart disorders, diabetes mellitus, liver cirrhosis Child A, essential hypertension, moderately severe chronic renal failure; $\mathrm{RR}=$ respiratory rate; $\mathrm{HR}=$ heart rate; $\mathrm{MAP}=$ mean arterial pressure; $\mathrm{ICU}=$ intensive care unit; LOS $=$ length of stay; $*=$ toxic-mediated ARDS ( 3 patients), near drowning ( 1 patient); $a=$ LOS in NIPSV success-patients vs. ETMV control-patients; $b=$ LOS in all NIPSV patients vs. ETMV control patients. 
6 patients in the ETMV group. The maximum PEEP applied during the trial was on average $6 \pm$ $0.8 \mathrm{~cm} \mathrm{H}_{2} \mathrm{O}$ for the NIPSV group compared with $10 \pm 2.5 \mathrm{~cm} \mathrm{H}_{2} \mathrm{O}$ in the ETMV group $(\mathrm{p}=0.001)$. There were no differences between the two groups in sex ratios, incidence of assumed pneumonia as a cause of ARDS, chronic underlying comorbidities, respiratory rate, heart rate and blood pressure, $\mathrm{PaO}_{2}, \mathrm{PaCO}_{2}$ or $\mathrm{pH}$ before institution of ventilation.

NIPSV failed in 4 patients $(33 \%)$ who then required EI. In these patients, the average duration of NIPSV before intubation was 6 hours (range 1-39 hours). The reasons for intubation were worsening of gas exchange with severe tachypnea in all 4 patients, complicated by haemodynamic instability in two. The overall success rate for the NIPSV trials was $66 \%$ (8 of 12). In the non-invasive ventilation group, intubated patients tended to be older and displayed at admission a higher SAPS II score than non-intubated patients (intubated patients: $51 \pm 14$ vs $38 \pm 14 ; p=0.15$ ). Moreover, they progressed to extrapulmonary organ failures and had positive blood cultures (Streptococcus pneumoniae in 3 patients, Klebsiella pneumoniae in 1 patient), contrasting with the absence of bacteremia in non-intubated patients $(p=0.07)$. At the moment of institution of ventilation, respiratory rate $(\mathrm{RR}), \mathrm{PaO}_{2}, \mathrm{pH}, \mathrm{Pa}-$ $\mathrm{CO}_{2}$ and $\mathrm{PaO}_{2} / \mathrm{FiO}_{2}$ did not differ significantly in either subgroup. As table 1 shows, there was a significant difference in the ICU length of stay between NIPSV success patients and ETMV controls $(p=0.004)$. Overall ICU mortality rate in the NIPSV group was $42 \%$ (25\% in the ETMV group; $p=0.69)$, with 2 ICU deaths unrelated to the lung injury, occurring once gas exchange finally improved and a respiratory steady state was achieved; causes of death were haemorrhagic stroke and acute myocardial infarction. The cumulative time spent on ventilation without discontinuation was shorter in the 12 NIPSV patients than in the ETMV controls $(74 \pm 68$ vs. $330 \pm 243$ $\mathrm{h} ; p=0.001$; figure 2). Arterial hypoxemia as assessed by the $\mathrm{PaO}_{2} / \mathrm{FiO}_{2}$ ratio, improved from baseline over time (60 minutes) in the NIPSV group (104 \pm 42 vs $146 \pm 52 \mathrm{mmHg}$ vs, $p=0.03)$, contrasting with a non-significant change in the ETMV group $(96 \pm 42$ vs. $109 \pm 34 \mathrm{mmHg} ; p=$ $0.39)$. As shown in figure 1 , the $\mathrm{PaO}_{2} / \mathrm{FiO}_{2}$ ratio was significantly higher in the NIPSV group after 1 hour of ventilation $(p=0.05)$. There were no differences in the time course of $\mathrm{PaCO}_{2}$ and $\mathrm{pH}$ within the NIPSV or ETMV groups. Causes of death for those NIPSV patients who required intubation included severe sepsis and multiple organ dysfunction in 3 patients. Cause of death in the control group was multiple organ dysfunction in 3 patients. The mask intolerance rate per se was extremely low and did not warrant switching NIPSV to endotracheal intubation. Observed complications in both groups are listed in table 2. The rate of serious complications showed a tendency to be higher among patients in the ETMV group than in the NIPSV group $(p=0.07)$.

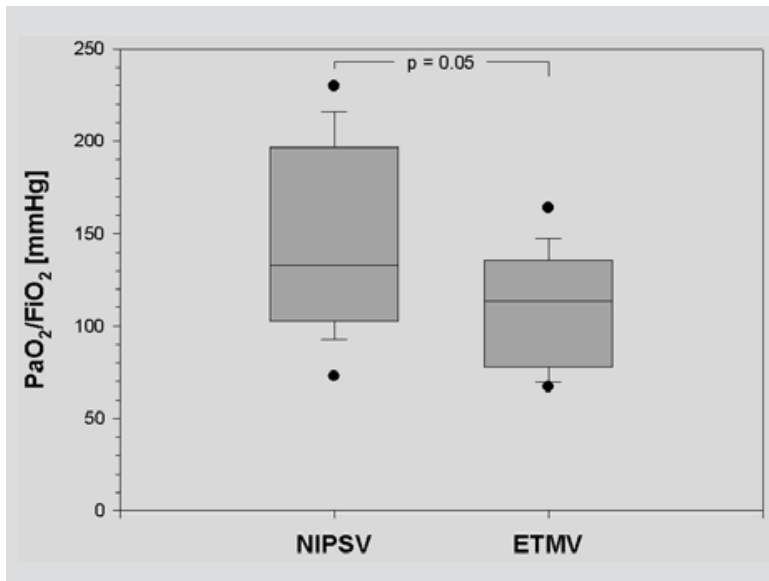

Fig. 1. - Comparison of the $\mathrm{PaO}_{2} / \mathrm{FiO}_{2}$ ratio between NIPSV and ETMV patients after 60' of ventilation. The horizontal box line represents the median; the box encompasses the $25^{\text {th }}-75^{\text {th }}$ percentiles and the errors bars show the $10^{\text {th }}-90^{\text {th }}$ percentiles. Filled circles represent outliers (erratic values). NIPSV = noninvasive pressure support ventilation; ETMV = endotracheal mechanical ventilation.

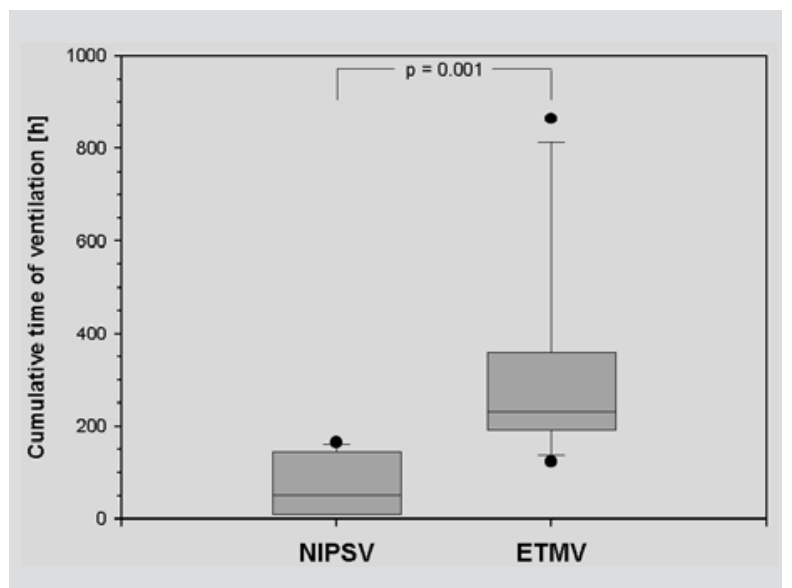

Fig. 2. - Comparison of the cumulative time spent on ventilation (without discontinuation) between the 12 non-invasively ventilated patients and the ETMV controls. The horizontal box line represents the median; the box encompasses the $25^{\text {th- }} 75^{\text {th }}$ percentiles and the error bars show the 10th-90th percentiles. Filled circles represent outliers (erratic values). NIPSV $=$ noninvasive pressure support ventilation; ETMV = endotracheal mechanical ventilation.

Table 2. - Observed complications in both groups

\begin{tabular}{lccc}
\hline VARIABLE & $\begin{array}{c}\text { NIPSV } \\
(\mathbf{n}=\mathbf{1 2})\end{array}$ & $\begin{array}{c}\text { ETMV } \\
(\mathbf{n}=\mathbf{1 2})\end{array}$ & p-value \\
\hline $\begin{array}{l}\text { Patients with complications } \\
\text { causing death in ICU }\end{array}$ & 5 & 3 & 0.38 \\
Myocardial infarction & 1 & 0 & 0.30 \\
Serious arrhythmias a & 1 & 3 & 0.27 \\
VAP & 0 & 3 & 0.06 \\
CIP & 0 & 3 & 0.06 \\
Intracranial hemorrhage & 1 & 0 & 0.30 \\
Severe sepsis & 4 & 5 & 0.67 \\
Nasal bridge injury & 2 & 0 & 0.13
\end{tabular}

VAP = ventilator-associated pneumonia; CIP = critically ill polyneuromyopathy. $\mathrm{a}=$ atrial fibrillation with rapid ventricular response, 2 patients; ventricular tachycardia, 2 patients. 


\section{Discussion}

This study shows that in stable non-immunocompromised patients meeting the definition of a primary ARDS and presenting at baseline or during the course of the disease without extrapulmonary organ dysfunction, NIPSV might be a feasible option. Compared to a matched group of similar patients treated with conventional invasive ventilation, NIPSV did not generate a high failure rate. Not surprisingly, avoidance of intubation was associated with a more rapid process of weaning and with a trend of lower frequency complications. The indication for NIPSV in non-COPD patients with severe acute hypoxemic respiratory failure has remained controversial for single pathologies such as ARDS [18]. As reported in a previous review, 185 ARDS cases from these mixed-patient populations have been considered, with an overall success rate (avoidance of intubation) of 58\% [11], a value fairly similar to ours (66\%). Ferrer et al., in a randomised clinical study, assessed the efficacy of non-invasive ventilation in 105 patients with severe AHRF, allocated to receive a face mask BiPAP or high-concentration oxygen therapy. In their study, causes of respiratory failure were mainly pneumonia and cardiogenic lung edema; however, 15 patients with ARDS were collected, seven in the BiPAP group and eight in the control group and the authors did not differentiate ARDS of primary or secondary origin. The efficacy of non-invasive ventilation was poor in the former ARDS subgroup, and all but one patient needed intubation [8]. The most important study distinguishing primary and secondary ARDS in addressing the question of the NIPSV impact on clinical outcome in patients with AHRF was published in 2001 by Antonelli et al. [7]. In this prospective, multicenter cohort study, the authors investigated potential factors involved in the failure. In this heterogeneous population, the overall efficacy of noninvasive ventilation in avoiding intubation (70\%) contrasted with the highest rate of failure observed in 86 patients fulfilling the diagnosis of ARDS (51\%); the rate of intubation was similar among patients with ARDS caused by a pulmonary or extrapulmonary condition, but sepsis on admission was associated, among other variables, with NIPSV failure [7]. Recently, the same group reported data from a large prospective investigation on non-invasive ventilation in ARDS patients [12]: in this study carried out by expert centres on NIPSV, this technique improved gas exchange and allowed avoidance of intubation in $54 \%$ of patients, who therefore had better outcomes than those who failed the non-invasive technique. The response to NIPSV was similar in patients with ARDS caused by pulmonary or extrapulmonary condition. Interestingly enough, the authors generated predictors of the need for intubation in their ARDS patients, which included greater age, higher SAPS II scores on admission and lower $(\leq 175)$ $\mathrm{PaO}_{2} / \mathrm{FiO}_{2}$ ratios after one hour of ventilation. These results, obtained in heterogeneous groups of ARDS-patients (the Authors considered primary and secondary ARDS-patients, the latter category in which the majority of the group found themselves), may not necessarily apply for selected ARDS-patients groups. By analysing possible predictors of NIPSV failure in our study, we found that all patients who needed intubation progressed to sepsis (positive blood cultures) and further to multiple organ dysfunction, contrasting with the absence of bacteremia and extrapulmonary organ failures in patients who had successful NIPSV. Although we closely monitored our patients and we used a low threshold for intubation, we cannot provide evidence that our stategy avoided dangerous delays in intubation in these patients. The results suggest firm avoidance of NIPSV as a first ventilatory support in ARDS patients demonstrating a clinical picture of extrapulmonary organ dysfunction at admission or in those developing a distant organ failure during the course of the disease. Baseline $\mathrm{PaO}_{2} / \mathrm{FiO}_{2}$ was quite low in both groups in our study. In contrast to the $\mathrm{PaO}_{2} / \mathrm{FiO}_{2}$ pattern observed in the ETMV group (figure 1), oxygenation improved more significantly at one hour in the group of patients treated with NIPSV, even with lower PEEP levels applied in this group than in the ETMV group. This evolution may be surprising and contrasts with the effects of the PEEP response reported in ARDS patients with a primary pulmonary injury [19]. Recent studies, however, indicate that promoting spontaneous ventilation in acute lung injury or ARDS may improve gas exchange by recruitment of non-aerated areas in the dependent lung zones through an increase of transpulmonary pressure [20]. We can therefore speculate that spontaneous ventilation per se might improve gas exchange more than a standardised volume-controlled ventilation in a fully sedated and relaxed patient, even in the presence of less conventional PEEP levels. Overall the ICU mortality rate tended to be higher in the NIPSV group. Although this value was influenced by two deaths unrelated to the lung injury itself, one cannot exclude a relationship between the non-invasive ventilatory treatment and the unsatisfactory outcome. The rate of serious complications demonstrated a trend towards being higher in the control group in this study. These complications involved mainly ventilator-associated pneumonia and critically ill polyneuromyopathy, two severe typical and relatively frequent complications observed during long term endotracheal mechanical ventilation $[16,21]$.

The present study has important shortcomings. The first limitation is the size of the population, even though studies on NIPSV in ARDS have included only a limited number of patients, except in Hilbert's study and Antonelli's two studies [3, 7, 12]. Moreover, our ARDS group was highly selected; consequently, the fairly good success rate of NIPSV could have arisen from a technique applied unexpectedly to a homogeneous group of haemodynamically stable and collaborative patients, most without ensuing multiple organ failures. In this setting, a successfully applied NIPSV in ARDS patients may represent an indicator of a low severity of illness. Finally, a case-control 
study may be biased toward an overestimation of the positive effects in the treatment group, for example through an intentional initial patient selection [22]. Even though we used a careful matching process allowing a good concordance of the matching criteria between the two groups (table 1) in order to moderate the risk of discrepancies in severity between cases and controls, the general nature of historical controls prevents any very careful matching with a prospective cohort. Our to some extent encouraging results coupled with the previous cited limitations, suggest that the effects of NIPSV in stable homogeneous subgroups of ARDS patients merit further investigations in randomised studies. Until additional data becomes available, a rather conservative approach should be taken, particularly in centres which are not expert on NIPSV.

\section{References}

1. Antonelli M, Conti G, Rocco M, et al. A comparison of noninvasive positive-pressure ventilation and conventional mechanical ventilation in patients with acute respiratory failure. New Engl J Med 1998; 339: 429-435.

2. Confalonieri M, Potena A, Carbone G, Porta RD, Tolley EA, Meduri GU. Acute respiratory failure in patients with severe community-acquired pneumonia: a prospective randomized evaluation of noninvasive ventilation. Am J Respir Crit Care Med 1999; 160: 15851591.

3. Hilbert G, Gruson D, Vargas F, et al. Noninvasive ventilation in immunosuppressed patients with pulmonary infiltrates, fever, and acute respiratory failure. $N$ Engl $J$ Med 2001; 344: 481-487.

4. Masip J, Betbese AJ, Paez J, et al. Noninvasive pressure support ventilation versus conventional oxygen therapy in acute cardiogenic pulmonary edema: a randomized trial. Lancet 2000; 356: 2126-2132.

5. Domenighetti G, Gayer R, Gentilini R. Noninvasive pressure support ventilation in non-COPD patients with acute cardiogenic pulmonary edema and severe community-acquired pneumonia: acute effects and outcome. Intensive Care Med 2002; 28: 1226-1232.

6. Jolliet P, Abajo B, Pasquina P, Chevrolet JC. Noninvasive pressure support ventilation in severe communityacquired pneumonia. Intensive Care Med 2001; 27: 812-821.

7. Antonelli M, Conti G, Moro ML, et al. Predictors of failure of noninvasive positive pressure ventilation in patients with acute hypoxemic respiratory failure: a multi-center study. Intensive Care Med 2001; 27: 17181728.

8. Ferrer M, Esquinas A, Leon M, Gonzalez G, Alarcon A, Torres A. Noninvasive ventilation in severe hypoxemic respiratory failure. A randomized clinical trial. Am J Respir Crit Care Med 2003; 168: 1438-1444.

9. Rocker GM, Mackenzie MG, Williams B, Logan PM. Noninvasive pressure support ventilation: successful outcome in patients with acute lung injury/ARDS. Chest 1999; 115: 173-17.

10. Bernard GR, Artigas A, Brigham KL, et al. Report of the American-European consensus conference on ARDS: definitions, mechanisms, relevant outcomes and clinical trial coordination. Intensive Care Med 1994; 20: 225-232.

11. Antonelli M, Pennisi MA, Conti G. New advances in the use of noninvasive ventilation for acute hypoxaemic respiratory failure. Eur Respir J 2003; 22: Suppl. 42, $65 \mathrm{~s}-71 \mathrm{~s}$.

12. Antonelli M, Conti G, Esquinas A, et al. A multiplecenter survey on the use in clinical practice of noninvasive ventilation as a first-line intervention for acute respiratory distress syndrome. Crit Care Med 2007; 35: $18-25$.

13. American Thoracic Society. Guidelines for the management of adults with community-acquired pneumonia: diagnosis, assessment of severity, antimicrobial therapy and prevention. Am J Respir Crit Care Med 2001; 163: 1730-1754.

14. American Thoracic Society Documents. Guidelines for the management of adults with hospital-acquired, ventilator-associated, and healthcare-associated pneumonia. Am J Respir Crit Care Med 2005; 171: 388-416.

15. Brochard L, Mancebo J, Wysocki M, et al. Noninvasive ventilation for acute exacerbation of chronic obstructive pulmonary disease. N Engl J Med 1995; 333: 817-822.

16. The Acute Respiratory Distress Syndrome Network. Ventilation with lower tidal volumes as compared with traditional tidal volumes for acute lung injury and the acute respiratory distress syndrome. $N$ Engl J Med 2000; 342: 1301-1308.

17. Perren A, Domenighetti G, Mauri S, Genini F, Vizzardi N. Protocol-directed weaning from mechanical ventilation: clinical outcome in patients randomized for a 30 -min or 120-min trial with pressure support ventilation. Intensive Care Med 2002; 28: 1058-1063.

18. Peter JV, Moran JL, Phillips-Hughes J. Noninvasive ventilation in acute respiratory failure. A meta-analysis update. Crit Care Med 2002; 30: 555-562.

19. Gattinoni L, Pelosi P, Suter PM, Pedoto A, Vercesi P, Lissoni A. Acute respiratory distress syndrome caused by pulmonary and extrapulmonary disease: different syndromes? Am J Respir Crit Care Med 1998; 158: 3-11.

20. Putensen C, Hering R, Wrigge H. Controlled versus assisted mechanical ventilation. Curr Opin Crit Care 2002; 8: 51-57.

21. De Jonghe B, Sharshar T, Lefaucheur JP et al. Paresis acquired in the intensive care unit: a prospective multicenter study. JAMA 2002; 288: 2859-2867.

22. Sacks H, Chalmers TC, Smith H Jr. Randomized versus historical controls for clinical trials. Am J Med 1982; 72: $233-240$. 